\title{
Complete heart block and bowel infarction secondary to rheumatoid disease
}

\author{
MARGARET WILSHER, ${ }^{1}$ W M IAN SMEETON ${ }^{2}$ TIMOTHY D KOELMEYER, \\ AND ANTONY H G ROCHE
}

From the ${ }^{1}$ Department of Cardiology, Green Lane Hospital, and the ${ }^{2}$ Department of Pathology, School of Medicine, Auckland, New Zealand

SUMMARY A case of complete heart block secondary to rheumatoid involvement of the conduction system is presented. The same patient died after infarction of the colon as a consequence of arterial spasm superimposed upon pre-existing rheumatoid changes in the mesenteric arteries. The coexistence of rheumatoid involvement of the heart and of the bowel vasculature has not previously been reported.

Key words: arthritis, arrhythmia, mesenteric artery, rheumatoid nodule, vasculitis.

Cardiac arrhythmias complicating rheumatoid arthritis are uncommon, but well reported..$^{1-12}$ However, in only a few instances have detailed studies of the conduction tissues been performed. 6-11 Ischaemic changes in the alimentary tract secondary to involvement of mesenteric arteries by rheumatoid disease are rare. We present a case of a patient with complete heart block due to rheumatoid disease, who died after infarction of the colon as a consequence of arterial spasm superimposed upon pre-existing rheumatoid changes in the mesenteric arteries.

\section{Case report}

The patient, a 59-year-old Caucasian male, had a 15-year history of seropositive erosive arthritis, involving predominantly hands, feet, elbows, and shoulders. Although deformity was minimal, pain and stiffness, particularly in the upper limb joints, restricted him to a sedentary lifestyle. Extraarticular manifestations included rheumatoid nodules, and during the year before admission to this hospital he had developed hepatosplenomegaly,

Accepted for publication 8 January 1985.

Correspondence to $\mathrm{Dr} M$ Wilsher, Chest Medical Unit, Green Lane Hospital, Auckland 3, New Zealand. lymphadenopathy, cutaneous vasculitis, - and a peripheral sensorimotor neuropathy. He had been treated with a variety of non-steroidal antiinflammatory drugs but had never received gold, penicillamine, or steroids.

Four months before admission he developed rapid atrial fibrillation and heart failure, and subsequently atrioventricular block appeared. He was admitted electively for pacemaker insertion.

On examination the patient was pale and cachexic. Pertinent cardiac findings included a bradycardia of 40 beats $/ \mathrm{min}$, supine blood pressure of 120/80 $\mathrm{mmHg}$, raised jugular venous pressure, mild cardiomegaly, and a soft ejection systolic murmur at the left sternal edge. There was no peripheral oedema, and the lung bases were clear.

The electrocardiogram (ECG) showed atrial fibrillation with complete atrioventricular block and an idioventricular rhythm of 40 beats $/ \mathrm{min}$. Radiography of the chest disclosed mild cardiomegaly. The erythrocyte sedimentation rate (ESR) was raised to $116 \mathrm{~mm} / \mathrm{h}$, and there was an anaemia (haemoglobin $9.8 \mathrm{~g} / \mathrm{dl}$ ) in the presence of normal iron stores. There was no abnormality of the leucocyte or platelet count. Renal function was normal, but there was a mild derangement of the liver enzymes. The rheumatoid factor was positive to a titre of 1:1024 (Rose-Waaler). 
Before the pacemaker could be inserted the patient collapsed in asystolic arrest. He was resuscitated, and a temporary transvenous pacemaker was inserted. Blood pressure was restored, though there was clinical and radiological evidence of severe left ventricular failure. Five hours later the patient developed severe central abdominal pain and watery diarrhoea.

Laparotomy was performed based on a clinical diagnosis of bowel ischaemia secondary to mesenteric artery embolus. The entire small and large bowel was found to be ischaemic but not gangrenous. There were no pulsations in the mesenteric arteries. The extent of ischaemia could be explained on the basis of a single embolus. A Fogarty catheter passed down the main right colic and major ileocolic arteries to the abdominal aorta without meeting an obstruction. On withdrawal of the catheter blood pulsated from each vessel, only to slow and finally stop within a minute. Marked arterial spasm was presumed and an infusion of nitroprusside was

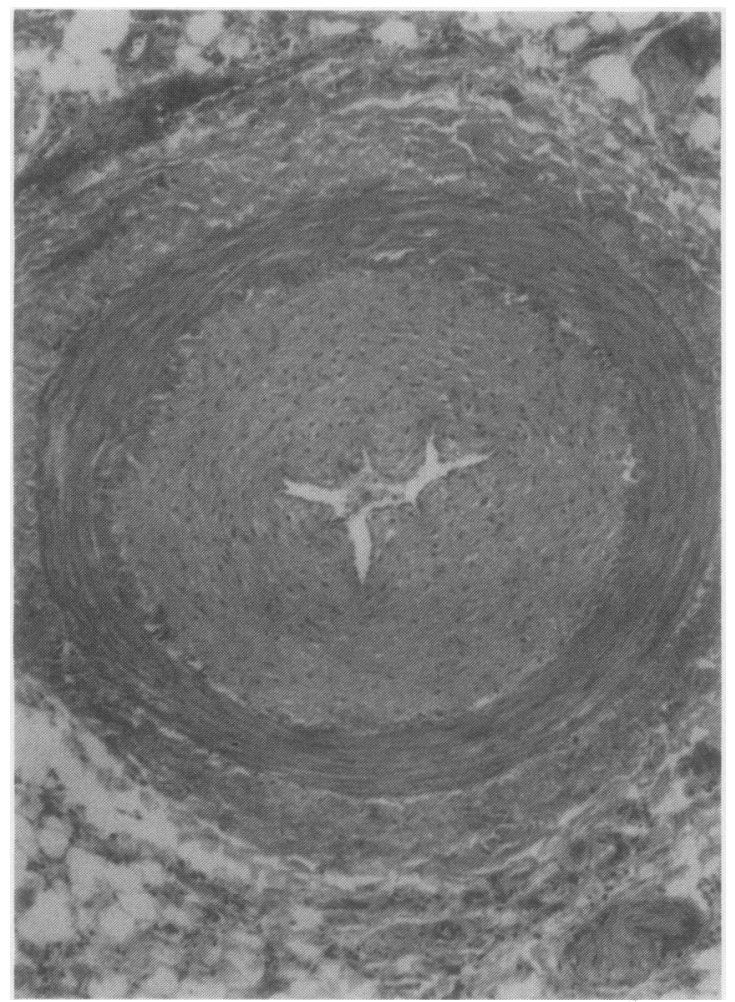

Fig. 1 A histological section of a distal mesenteric artery demonstrating the marked intimal hyperplasia. (Haemotoxylin and eosin, $\times 80$ ).

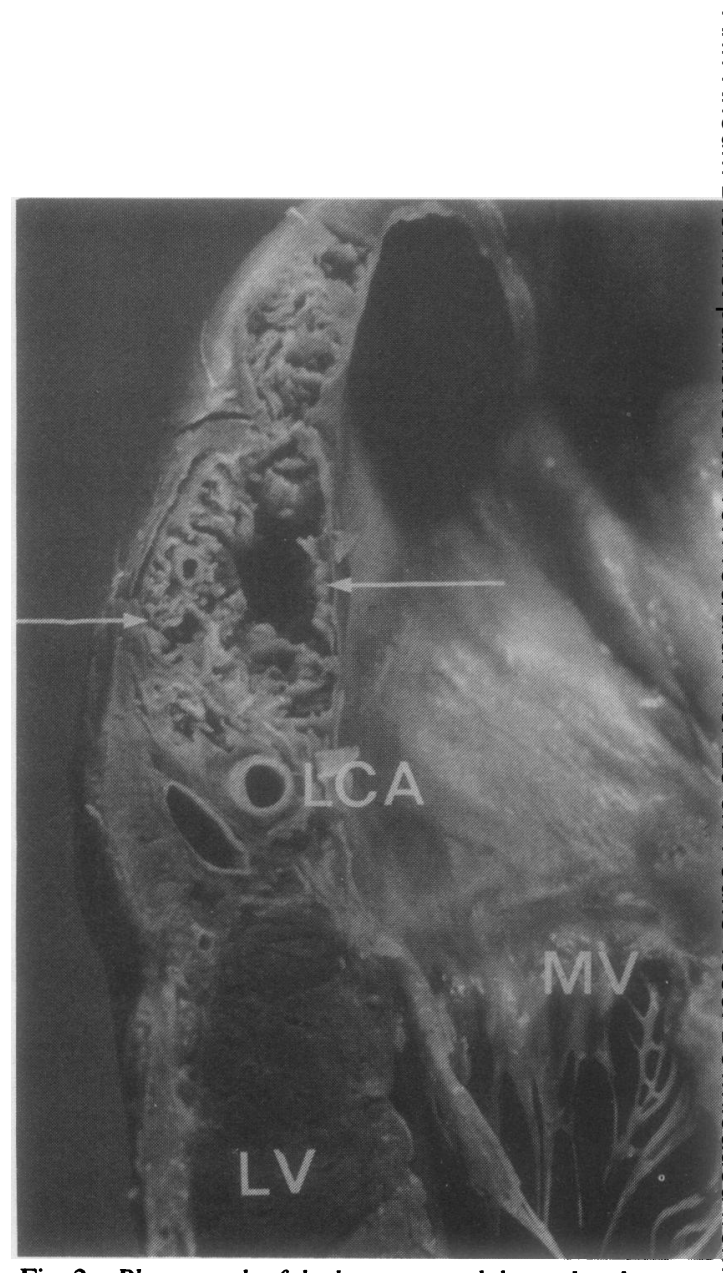

Fig. 2 Photograph of the heart opened down the obtuse margin showing the necrotic nodule (arrows) within the left atrial wall. $M V=$ mitral valve; $L C A=$ left circumflex artery; $L V=$ left ventricular wall.

commenced at a rate of $33 \mu \mathrm{g} / \mathrm{min}$. Almost im- $\frac{\hat{\partial}}{3}$ mediately there was an improvement in the colour of the bowel, with restoration of arterial pulsations? and return of peristaltic activity. The operative $\frac{D}{2}$ procedure was concluded, and the patient returned to the intensive care unit on a nitroprusside infusion, $N$ but circulatory collapse ensued and the patient died 12 hours after surgery.

The autopsy findings were as follows:

(1) Bowel. The colon was gangrenous throughout its entire length. The small bowel, though congested, $\frac{\mathbb{C}}{\mathbb{D}}$ was not infarcted and appeared viable. The major $\stackrel{9}{?}$ mesenteric vessels were free of thrombotic or 0 embolic material. Histology of mesenteric arteries taken from both superior and inferior mesenteric $\stackrel{\mathbb{Q}}{\mathbb{Q}}$ territories close to the bowel wall showed marked $\vec{\Phi}$ intimal proliferation but no evidence of active $\frac{\varrho}{\sigma}$ vasculitis (Fig. 1). 
(2) Heart. There were loose pericardial adhesions. The heart weighed $430 \mathrm{~g}$. The coronary arteries showed minimal atherosclerosis. The atria were mildly dilated, and the atrial walls were diffusely thickened and rigid in texture. The posterior left atrial wall contained a necrotic nodule $1.5 \mathrm{~cm}$ in diameter (Fig. 2). The ventricular chambers were normal in size, and the ventricular myocardium was free of scarring or necrosis. The heart valves were normal.

Histologically, much of the atrial muscle including the sinus node was replaced by loose fibrous tissue containing a sparse focal chronic inflammatory infiltrate. Occasional small foci of fibrinoid necrosis with surrounding pallisading of histiocytes were present. In the nodule shown in Fig. 2 there was

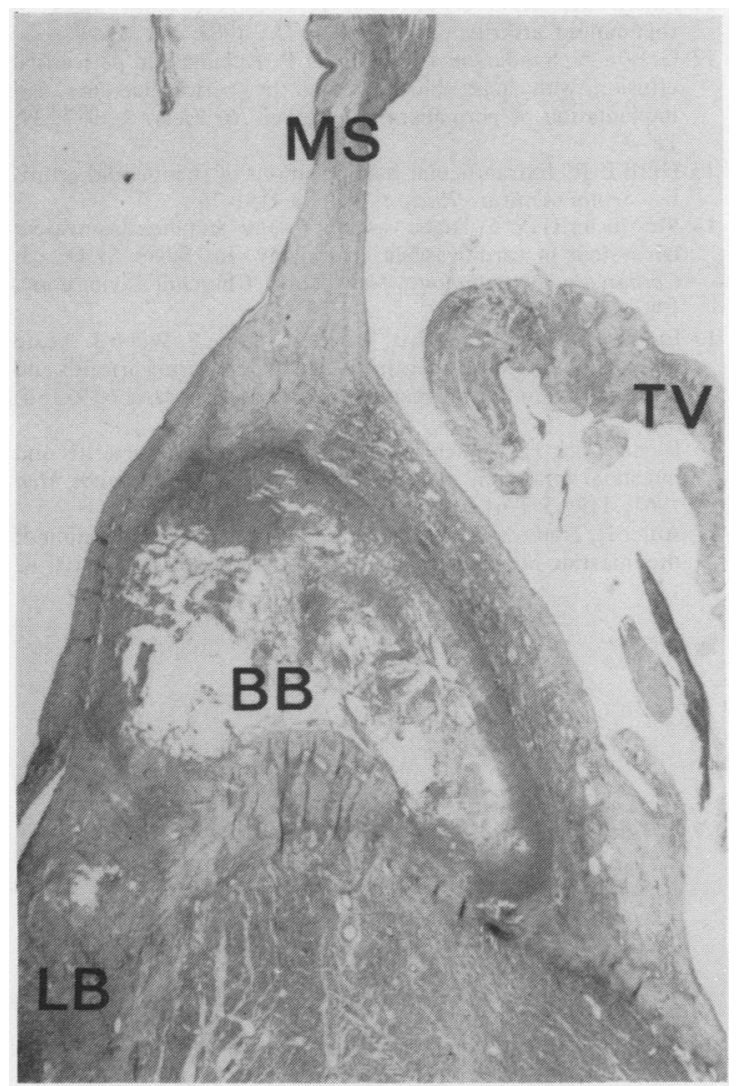

Fig. 3 Histological section through the membranous septum (MS) down into muscular interventricular septum. The region of the branching bundle $(B B)$ has been destroyed by inflammatory tissue which extends down into the proximal left bundle branch $(L B)$. TV =tricuspid valve. (Haematoxylin and eosin, $\times 10$ ). tissue necrosis, with the inflammatory infiltrate containing a prominent neutrophilic component.

The atrioventricular conduction tissues were serially sectioned at $10 \mu \mathrm{m}$ with every 20 th section stained. Dense chronic inflammation with central cavitation replaced the atrioventricular node. The inflammatory involvement followed the penetrating bundle (His bundle) as it passed through the central fibrous body to reach the crest of the interventricular septum (Fig. 3) to become the branching bundle and then further involved the proximal portion of the left bundle branch. Elsewhere the ventricular myocardium contained only a few sparse occasional lymphocytic aggregates. The coronary arteries were not inflamed, though occasional intramyocardial arteries displayed marked intimal thickening, a feature particularly prominent in the atrioventricular node artery.

\section{Discussion}

Rheumatoid heart disease may take several forms. Although specific for the condition, cardiac rheumatoid nodules are found in only $1-3 \%$ of patients with rheumatoid arthritis who come to autopsy. These granulomas have been reported in the pericardium, epicardium, myocardium, endocardium, all valve cusps and rings (predominantly mitral and aortic), and chordae tendinae. Other non-specific types of cardiac lesions, however, have been observed at necropsy in these patients, including pericarditis, myocarditis, coronary arteritis, and patchy valvular fibrosis. ${ }^{1314}$ Conduction disturbances have been described in association with rheumatoid percarditis and valvular heart disease. ${ }^{712}$

There are a number of mechanisms by which the atrioventricular conduction system can be involved in rheumatoid arthritis. These include direct involvement by granulomas or by extension of the granulomatous inflammation from the base of the aortic or mitral valve. In one case no active granulomas were present in the conduction system, but fibrotic patches were present in the areas where presumably active granulomas had been present. ${ }^{10}$

There is a range of vascular lesions occurring in rheumatoid arthritis and these may be encountered singly or in combination. These changes include intimal proliferation (most often involving the digital arteries), necrotising arteritis of both small and medium sized arteries (indistinguishable from polyarteritis nodosa), a subacute arteritis (where the involved vessel is infiltrated with mononuclear cells), and a leucoclastic vasculitis of the venules and capillaries in the skin. ${ }^{14}$ As a result of these vascular changes a variety of clinical features have been described, which include skin rashes, cutaneous 
ulceration, peripheral gangrene, peripheral neuropathy, and rarely, visceral lesions.

Involvement of small arteries can cause multiple ischaemic ulcers of the intestine, resulting in haemorrhage or perforation. ${ }^{15}$ Involvement of larger arteries may cause segmental (or more extensive) bowel gangrene. ${ }^{1617}$ Perisplenitis and splenic infarcts are a well known cause of left hypochondrial pain in rheumatoid patients, and acute arteritis of hepatic, cystic, and pancreatic and renal vessels has been reported. ${ }^{15}$

A remarkable feature of our case was the intense spasm of the mesenteric arteries seen at laparotomy, possibly precipitated by the previous arrest. Also, rather than the acute arteritis described in other reported cases, the lesion in the mesenteric arteries seen here was intimal proliferation. To our knowledge this has not been described before. Patients who develop conduction disturbances generally have severe rheumatoid disease, often with extraarticular manifestations or high rheumatoid factor titres. ${ }^{11}$ Our patient probably had systemic vasculitis as shown by cutaneous vasculitis and peripheral neuropathy, and it is possible that mesenteric arteritis preceded the development of intimal proliferation. We are not aware of any reports in the literature of the coexistence of rheumatoid involvement of the heart and of the bowel vasculature.

We are grateful for the assistance of Dr A C Hayton, Physician, Taranaki Base Hospital and Dr W Tangi, Surgical Registrar, Auckland Hospital.

\section{References}

1 Handforth C P, Woodbury J F L. Cardiovascular manifestations of rheumatoid arthritis. Can Med Assoc J 1959; 80: 86-90.
2 Gowans J D C. Complete heart block with Stokes-Adams $\frac{\square}{\Omega}$ syndrome due to rheumatoid heart disease. N Engl J Med 1960; $\because$ 262: 1012-4.

3 Lebowitz W B. The heart in rheumatoid arthritis (rheumatoid disease). A clinical and pathological study of sixty-two cases. Ann Intern Med 1963; 58: 102-23.

4 Hoffman F G, Leight L. Complete atrioventricular block $\overline{\bar{c}}$ associated with rheumatoid disease. Am J Cardiol 1965; 16: 585-92.

5 Gallagher P J, Gresham G A. Heart block with infected cardiac』 rheumatoid granulomas. Br Heart $J$ 1973; 35: 110-2.

6 Sobin L H, Hagsom J W C. Lesions of cardiac conduction tissue $\vec{\circ}$ in rheumatoid arthritis. JAMA 1962; 180: 1-5.

7 Carpenter D F, Golden A, Roberts W B. Quadrivalvular $\vec{\omega}$ rheumatoid heart disease associated with left bundle branch block. Am J Med 1967; 43: 922-9.

8 Kirk J, Cosh J. The pericarditis of rheumatoid arthritis. $Q$ J. Med 1969; 38: 397-423.

9 Harris M. Rheumatoid heart disease with complete heart block. J Clin Pathol 1970; 23: 623-6.

10 Lev M, Bharati S, Hoffman F G, Leight L. The conduction system in rheumatoid arthritis with complete atrioventricular block. Am Heart J 1975; 90: 78-83.

11 Ahern M, Lever J V, Cosh J. Complete heart block in rheumatoid arthritis. Ann Rheum Dis 1983; 42: 389-97.

12 Gelson A, Sanderson J M, Carson P. Rheumatoid pericardial effusion with heart block treated by pericardiectomy and ${ }^{\mathbb{D}}$ implantation of permanent pacemaker. Br Heart J 1977; 39: $\vec{\bullet}$ 113-5.

13 Hurd E R. Extraarticular manifestations of rheumatoid arthri-. tis. Semin Arthritis Rheum 1979; 8: 151-76.

14 McAllister H A. Collagen vascular disease and the cardiovascular system in cardiovascular pathology. In: Silver M D, ed.S Cardiovascular pathology. New York: Churchill Livingstone, 1983; 2: 1005-33.

15 Lindsay M K, Tovadia H B, Whyte A S, Lee P, Webb J. Acute abdomen in rheumatoid arthritis due to necrotizing arteritis and intestinal infarction in rheumatoid disease. $\mathrm{Br}$ Med $J$ 1973; ii:Ō 592-3.

16 Bienenstock H, Minich R, Rogoff B. Mesenteric arteritis and intestinal infarction in rheumatoid disease. Arch Intern Med 1967; 119: 359-64.

17 Adler R, Norcross B M, Lockie L M. Arteritis and infarction of the intestine in rheumatoid arthritis. JAMA 1962; 180: 100-4.응 